\title{
Recommendation System: State of the Art Approach
}

\author{
Mohammad Aamir \\ PG Student \\ AKG Engineering College \\ Adhyatmik Nagar, GZB (UP) India
}

\author{
Mamta Bhusry \\ Professor \\ AKG Engineering College \\ Adhyatmik Nagar, GZB, (UP) India
}

\begin{abstract}
A Recommender System (RS) is a composition of software tools and machine learning techniques that provides valuable piece of advice for items or services chosen by a user. Recommender systems are currently useful in both the research and in the commercial areas. Numerous approaches have been proposed for providing recommendations. Certainly, recommendation systems have an assortment of properties that may entail experiences of user such as user preference, prediction accuracy, confidence, trust, etc. In this paper we present a categorical reassess of the field of recommender systems and Approaches for Evaluation of Recommendation System to propose the recommendation method that would further help to enhance opinion mining through recommendations.
\end{abstract}

\section{Keywords}

Recommender System, Filtering, Trust Based, Agent Based, Prediction

\section{INTRODUCTION}

Recommender systems are present in many multifarious applications so that the user can have a look at the colossal collection of items. The recommender system provides a catalogue of recommended items that are preferred by the user [3]. It alleviates the process of finding more favourable items in the collection through encouraging the users to decide on suitable items. Higher online transaction is one of the biggest effects of easier Internet usage in most of the countries. Nowadays, it is very common that people place the web as the source of information to buy or sell something. Therefore, there exist many online shops, in different forms, varying from website with private domain to thread in an online forum. This leads to both advantages and also disadvantages for customer in different ways [1]. The main advantage is that a customer has more options to buy. But simultaneously it can also be disadvantageous because with so many options, customer will find it difficult to choose one single product keeping in view various criteria e.g. which shop can be trusted, who has good customer service, and also who offers good price. Thus, the main problem is that there is no onestop place to search comprehensive information about online selling and buying. The required information related to online selling and buying includes list of products, list of online shops, and also a set of recommendations about choosing product and shop [2].

\section{RECOMMENDATION SYSTEM}

The success of online product stores has made thousands of products available on the websites of different organizations. Almost anything, ranging from a needle to high end digital devices can be ordered online. Thus the customer is overloaded with huge amount of information about in numerous products and services [4]. Traditionally, users get a word of mouth recommendation for a product, and they ask for the recommended product at the shop. A similar analogy works for library and information services because people tend to buy books from bookstalls which are suggested to them by their faculty, friends and/or colleagues [5]. These days many online book stores are available which have a huge collection of material available with them in the form of ebooks, research papers and journals. A user cannot easily navigate through all of the contents available on these websites. Recommendations are different from a prediction. To make a prediction we require information even about rarely rated items whereas to make a recommendation we need to know only a subset of information [3]. Therefore recommendation system reduces the problem of large memory requirements and huge computation time. After collection of data it is analyzed and learned using various machine learning techniques like clustering and categorization.

\subsection{Examples of Recommendation system}

- The online book retailer INFIBEAM which endows an average rating of the displayed books by the user, and a catalogue of other books that are purchased by the users. At times the system displays a catalogue of additional items while downloading software that are preferred by the user.

- The online e-stores are developed to suggest items to the users based on their preferences and taste, thereby creating an automated and intelligent system to serve the purpose of word of mouth recommendations

- Online library stores are also similar to ecommerce stores as readers, publishers, buyers and sellers do activities like reading, publishing, buying and selling of various articles and search for various categories of books, research papers and journals on them.

\section{PROS AND CONS OF RECOMMENDER SYSTEM}

Some of the pros and cons of recommender systems are as follows.

\subsection{Pros}

- Based on actual user behavior that is user can make decision directly based on the results.

- Effective tools for Personalization

- Always up to date

\subsection{Cons}

- There is no one stop place to search comprehensive information.

- Changing Data

- Changing user preferences 


\section{LITERATURE REVIEW}

A massive quantum of research has been conducted in the field of recommender systems, which predominantly focuses on developing new algorithms to achieve preferable recommendations. The designer that designs an application has a collection of algorithms, and must make a pertinent decision for selecting an algorithm to achieve his goals; the selection process is solely based on experiments, in which the performance of numerous users recommendations are collated [5]. For the given structural constraints the designer can then plump for the best performing algorithm. Moreover, numerous researchers have suggested some new algorithms for the recommendation process in which the performances are compared by applying some assessment metric i.e. the prediction power of the recommendation system in order to provide a ranking of the candidate algorithms. Primarily most of the recommender system are assessed and ranked on their prediction potency. Prediction is an ability of the recommender system to precisely predict the choices of the user. Accurate predictions may play a vital role in the working of the recommender system but it is insufficient to set up a good recommendation engine. The success of a recommender system can be evaluated if the properties used are specified. Then only we can calculate the system performances based on those properties in context of a specific application.

Eivind Bjørkelund, et.al. [19] Described how opinion mining can be applicable on various web sites and also shows the visualization of sentiment analysis of textual reviews on Google. The techniques and prototype can be applied on adequately large data set containing sentiment data. It can be used on any opinionated data set feature search and extractions. Further, it can also be helpful in any temporal data set burst detection.
Li-Chen Cheng, et.al. [17] Proposed method that combines the overall rating to discover the relation of the features with the associative classification methodology. A framework is proposed to unearth those changes of opinion that can recognize the users' opinion about a product. To discover the trends of word of mouth (WOM) information for arbitrary online reviews.

Walter Kasper, et.al. [16] Described a system that collects comments from the hotel website and creates classified and structured overviews of such comments and facilitates access to that information. And these comments are important for quality control to the hotel management, too. Further the system is capable of detecting and retrieving reviews on the web, to classify and analyze them, as well as to generate comprehensive overviews of these comments.

\section{RECOMMENDATION PROCESS}

Generally, all recommendation systems adhere to a concrete process [6] to produce product recommendations, which is shown in figure 1 .

Based on the used information, different approaches of the recommender system can be disintegrated. The three information sources that are considered as input for the recommendation system are

(1) User generated data (example: age, gender)

(2) Types of item data (example: tags...)

(3) The user preference data (example: ratings)

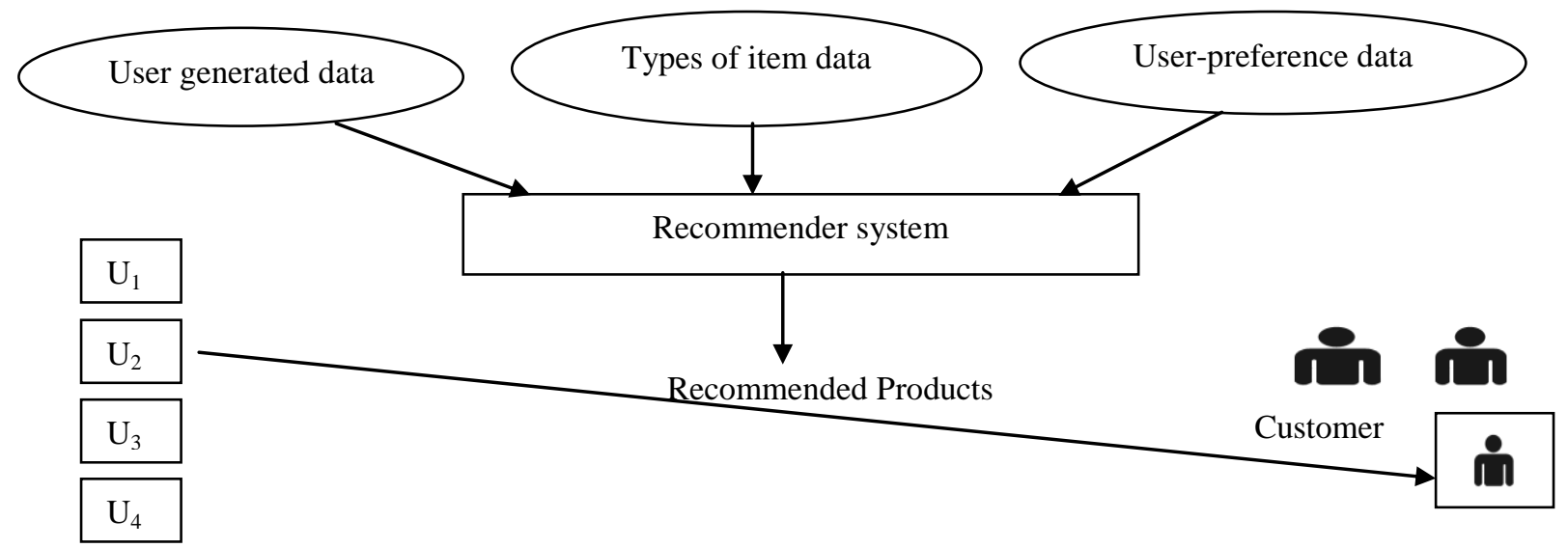

Products

Fig. 1. Framework of Recommendation Process

\subsection{Approaches of Recommender Systems}

Recommender Systems (RS) are for those individuals who do not have sufficient personal skill or expertise to analyze the profuse number of different items that an online web site may offer [7]. Popular online websites employ RS to meet the requirement of every customer in the store. Usually recommendations are done to meet the requirement for diverse users or cluster of users that receive multifarious suggestions. Personalized recommendation systems try to foretell the most appropriate products or services depending on the user's preferences. Moreover, they also offer non- personalized recommendations which are more manageable to procreate. Recommender systems can be used by diverse applications like E-commerce and can be categorized in two ways:

(i) Personalized recommender system

(ii) Non-personalized recommender system.

Personalized recommender systems are the one that are mostly used by online web sites to recommend products to their customers depending upon user preferences and constraints. The prediction of the future buying behaviour of 
the customer can be done by analyzing the previous buying behaviour of the customer.

Non-personalized recommender systems recommend products to the customer based on reviews of customers about the products. These systems require minor customer attainment to generate the recommendations as they are automatic and transient. For example, Reel.com and Levis web sites are treated as non-personalized recommender systems.

\subsection{Recommendation System Properties}

\subsubsection{User preference}

User preference is an essential part of any recommender system. Recommendations may help user to explore and gather knowledge about the product more quickly and efficiently and not to crawl through hundreds or thousands of products. Users can see the recommended products based on their preferences on only one or two pages. Obviously, the recommended list of products which user gets may not provide all the required information but they are a hint for the user.

\subsubsection{Prediction Accuracy}

This is considered as the most important technique in the recommendation system. The prediction which is done by the prediction engine is at the end of the recommender system. The user opinions are predicted over items by this engine. For example, the engine predicts the rating of movies, books and other items. Users prefer those recommender systems that provide more accurate prediction.

\subsubsection{Confidence}

The level of systems trust in its recommendation or prediction is known as confidence in the recommendation system. Confidence in the predicted property rises as the amount of data rises. However the system assigns confidence scores to an item which in turn is very helpful for the user while accessing the list of items.

The user is likely to go for further research before making any decision if the system accounts a little confidence in a recommended item. For example, if the system recommends two movies of five star ratings one with a very low confidence and another movie with high confidence then it might be possible that the user may attach the second movie directly to its watching list, but may further investigate regarding the first movie by reading its synopsis before watching it.

\subsubsection{Trust}

Trust refers the users trust in the recommendation system. The system may recommend few items in accordance to the user preference and likes. The user observes that the system recommended some reasonable items and this increases trust in the system recommendations for unknown items as well as interaction between system and user is the basis of building trust.

\section{FUNCTIONAL CLASSIFICATION OF RECOMMENDER SYSTEMS}

Recommender systems can be categorized into various categories based on the information they use to recommend items.

- Content-based Filtering Recommender Systems

- Collaborative Filtering Recommender System
- Demographic Filtering Recommender Systems

- Hybrid Recommender Systems

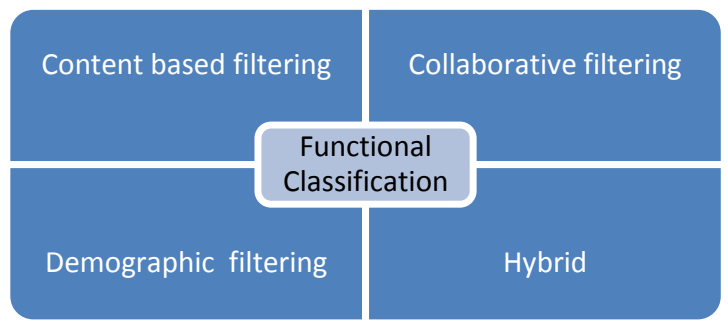

Fig. 2. Framework of functional classification for recommender system

\subsection{Content-based Filtering Recommender Systems}

Content-based filtering, also known as cognitive filtering, recommends items on the basis of comparison between the content of the items and a user profile [10]. Various issues have to be considered when executing a content-based filtering system. First, terms can either be allocated automatically or manually. When terms are allocated automatically, a method has to be carried out that can extricate these terms from items. Second, the terms have to be represented in such a way that both the user profile and the items can be collated in a significant way. Third, a learning algorithm has to be selected that should be able to learn the user profile based on the items and can make recommendations based on this user profile. Genetic algorithms, relevance feedback, Bayesian classifier and neural networks are among the learning techniques for learning a user profile.

\section{Advantages of content- based approach}

- Content-based methods have to only scrutinize the items and user profile for recommendation.

- Content-based method can enlighten the user on the features on which recommendation is based.

- New items can be recommended before being rated by an extensive number of users.

Disadvantages of content- based approach

- If the content do not include adequate amount of information to differentiate the items accurately, the recommendations received will be not be precise.

- The system does not provide reliable recommendation as there's is not adequate amount of information to construct a good profile of a user.

- Another drawback is the Serendipity problem in which the system will recommended or suggest those items who have high preferences when they are matched against the user profile; that is why user get recommended only those items or products that are similar to those that are already rated.

Serendipity and novelty are important for any recommendation system. Serendipity is the art of recommending an astonishing item to the user of which he is not already aware of and he might not have easily discovered it. Novelty in a recommendation system means that recommending an item of similar type to a user which he is already aware of but could not have found it himself. 


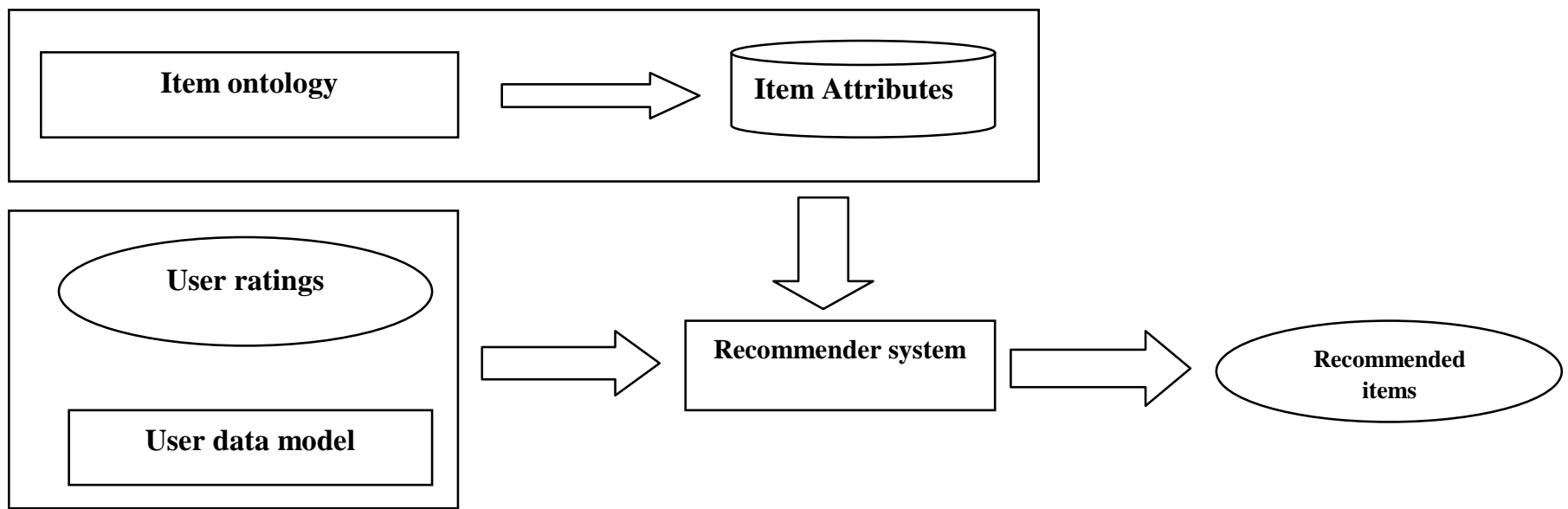

Fig. 3. Framework of Content-based approach

\subsection{Collaborative Filtering Recommender Systems}

This is a method of making automatic predictions about the interests of a user by accumulating preferences from many users [9].The purpose of collaborative filtering systems is to recommend new items to the user or envisage the utility of a certain item, based on user's previous likings and also on the opinions of other likeminded users.

It is extensively used and possibly the most well-known recommendation technique implemented in numerous eCommerce applications. Normally, collaborative filtering systems amass the ratings of all the items from the user preference list. Opinions can be classified explicitly and implicitly, the former is performed by the user as a rating score and later is derived from the historical data of the user.

The collaborative filtering can adapt with Neighborhood methods, as shown in figures 4(a) and 4(b).

(i) User-based collaborative Filtering:

This approach computes the correlation with all other users for each item and aggregate the rating of highly correlated users.

(ii) Item-based collaborative Filtering:
This approach computes for each user item the correlation with all other item and aggregates for each user the ratings for item that are already highly correlated.

Advantages of Collaborative Filtering system

- The system does not require a representation of items in terms of features but it is based only on the judgment of participating user community. For example songs, books, jokes, etc.

- As it does not require any kind of human involvement, the scalability of the items database is large.

- For prediction, they can use cross type recommendation which are different for different users. This does not require any kind of domain knowledge, hence saves times.

Disadvantages of Collaborative Filtering system

- For an item to get recommended to a user, either it should be rated by another user(s) or it is correlated with other similar items of the database

- In spite of having a large database for many e-commerce applications the active users rate only few items which leads to very sparse results

- The approach is expensive and time consuming due to the diversity of opinions of large number of users.

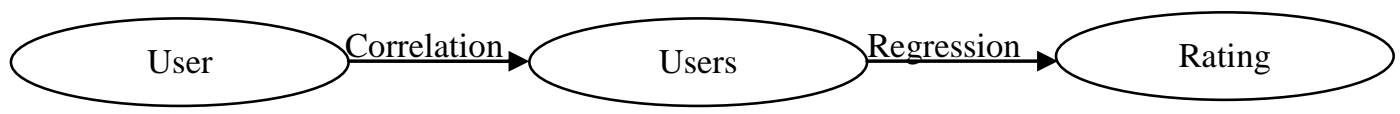

Fig 4(a): Framework for user based collaborative filtering

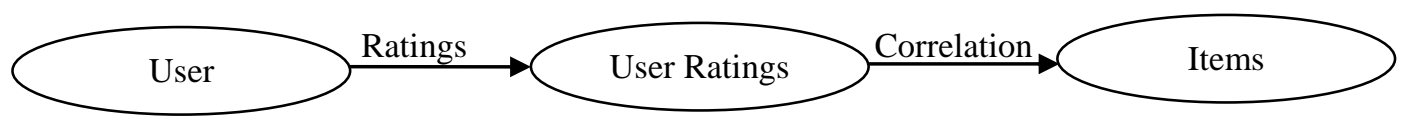

Fig 4(b): Framework of Item based Collaborative filtering

\subsection{Demographic Filtering Systems}

It is a method that utilizes user's attributes, classified as demographic data, in order to produce their recommendations such as age, gender, education, etc. of people for identifying types of users [10].The concept used in demographic recommender systems is that the descriptions of people are used to learn the relationships between a single item and the class or the kind of people who liked it. The basis of recommendation for demographic based recommender system uses previous knowledge on demographic information about the user and their opinions for the recommended items 
Demographic systems depend on the hypothesis that all users belonging to a certain demographic group have similar preferences or taste that is why demographic systems are stereotypical

Advantages of demographic filtering system

- The approach is easy and quick for generating results based on a small number of observations.

- Does not require the record of user ratings that are required by collaborative and content based techniques.

Disadvantages of demographic filtering system

- The collection of entire user information is impractical for efficient demographic filtering considering the security and privacy issues.
- Demographic filtering is largely based on users interests which lead the system recommend the same item to the users of same demographic profiles and results.

\subsection{Hybrid Recommender Systems}

A hybrid approach combines the content based filtering system and the collaborative filtering system. In this system it is also possible to use the recommendations of the two filtering techniques independently [9]. A hybrid recommender system is another category of recommender systems that tries to rise above the limitation of other approaches. As it combines two or more recommendation techniques to achieve better optimization. One of the most popular hybrid approaches is the one that adapt the heuristic combination of content based and collaborative filtering.

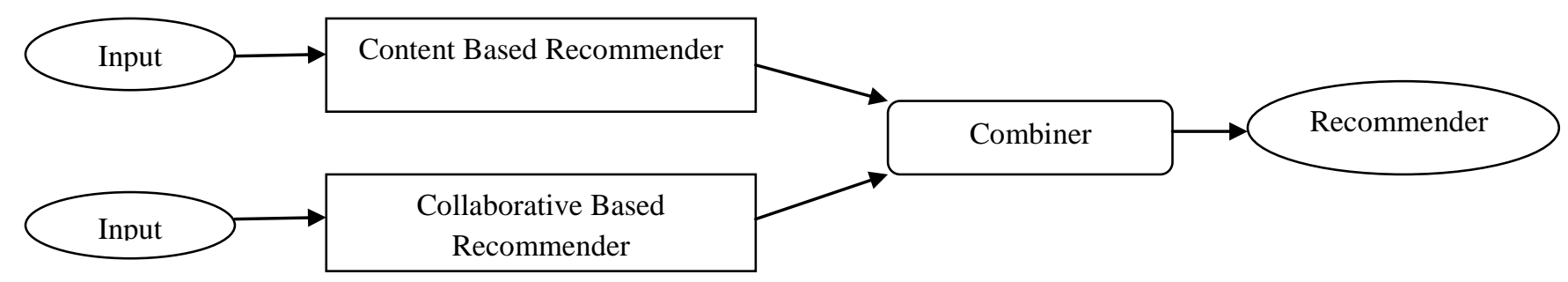

Fig. 5. Framework of Hybrid Recommendation

Various methods of hybridization are as discussed below:

Weighted: In this technique the scores of a recommended item is calculated from the outcome of all of the available recommendation techniques and are mixed with each other to manufacture a single recommendation

Switching: It is the process in which the system switches between recommendation techniques based on some yardsticks. This technique brings an surplus amount of complexity into the recommendation process.

Mixed: When recommendations of more than one technique are presented together to generate large number of recommendations simultaneously then the best feasible technique is to use the mixed hybrid recommendation.

Feature combination: The feature combination hybrid in this the number of users who have rated for an item experiences a reduced sensitivity of the system because the system considers collaborative data without relying on it exclusively. That is why different data sources are thrown together into a single recommendation algorithm.

Cascade: Unlike the other hybridization method cascade involves a process in which one recommender refines the recommendations given by another.

Feature Augmentation: The technique employed here is to manufacture a rating or classification of a product and that classification are then included into the processing of the next recommendation technique. That is the output from one technique act as an input feature for another.

Advantages of hybrid recommender system

- Hybridized system outperforms content based recommender and collaborative filtering recommender system, as the relative accuracy of the recommender is comparatively high.
- Cold start problem due to short user profiles and availability of sparse ratings of a user can be handled effectively by using hybrid recommendation system [12].

Disadvantages of hybrid recommender system

- It is complex to develop a hybridized recommender system

- It may also not feasible to develop all possible combinations of hybridized recommendation system [11].

\section{TRUST BASED SOCIAL RECOMMENDER SYSTEMS}

One can obtain information and generate more useful recommendation about user profiles and relationships between users from information obtained through social networking websites such as Facebook and LinkedIn. Its basic advantage is that the user ushers in more trust in these recommendations.

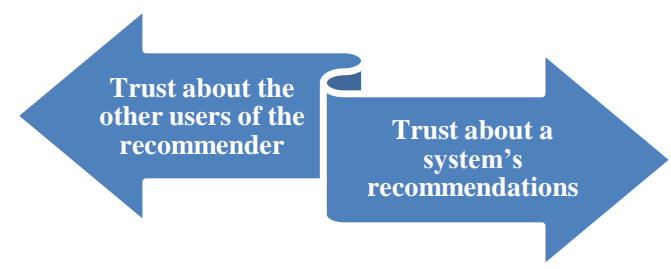

Fig. 6. Notions of trust

Out of the seven roles played by the RSs, trust emerges as one of the most important one [13]. 
Table 1. Roles of Recommender System

\begin{tabular}{|l|l|}
\hline \multicolumn{1}{|c|}{ Roles } & \multicolumn{1}{c|}{ Explanation } \\
\hline Effectiveness & $\begin{array}{l}\text { Good decisions can be taken with the } \\
\text { help of effectiveness by the user }\end{array}$ \\
\hline Satisfaction & $\begin{array}{l}\text { Ease of use or enjoyment can be } \\
\text { increased with the help of satisfaction }\end{array}$ \\
\hline Scruitability & $\begin{array}{l}\text { If it is wrong then the users have the } \\
\text { option to tell the system }\end{array}$ \\
\hline Persuasiveness & $\begin{array}{l}\text { Try and buy are the two convincing } \\
\text { powers of this feature }\end{array}$ \\
\hline Trust & $\begin{array}{l}\text { It increases the confidence level of } \\
\text { the user in the system }\end{array}$ \\
\hline Transparency & $\begin{array}{l}\text { Working of the system is explained } \\
\text { by implementing transparency }\end{array}$ \\
\hline Efficiency & $\begin{array}{l}\text { User can take decisions faster with } \\
\text { the help of efficiency }\end{array}$ \\
\hline
\end{tabular}

The vision on the inherent risk trustworthiness of each other that arises between the entities that mediate on-line transactions [14]. They also explained why traditional network security mechanisms are incomplete in their function to manage trust. To minimize the risk they insisted on trust management which becomes an essential component in distributed network environment. Using the definition of trust, considering the issues which motivate need for complementary trust management they proposed a trust computing model. This model extends and generalizes security and trust management emphasizing on the following four goals:

To adopt a decentralized approach to trust management.

- To generalize the notation of trust.

- To lessen ambiguity by making trust statements more explicit.

- To facilitate the exchange of trust -related information via a common protocol.

\section{AGENT BASED RECOMMENDER SYSTEMS}

The recommender system [15], in which agent's are used as an enabling technology are known as Agent Based Recommendation Systems. To use agents as recommenders will probably be the most efficient solution. However, evaluation algorithms and existing quality measures work in some finite domain only. For example, agents can check the syntactic and semantic validity of some information resources. Some research areas are crucial to both agent technologies and recommender systems; those are machine learning, user modelling and filtering techniques. For recommender systems, agent research offers ways to manage autonomy, pro-activity, distribution, reputation and trust, etc. For agent technologies, recommender systems offer a practical and important application domain with useful concepts [15]. Now, multiagent systems researches have been extensively studying the notions of reputation and trust, as they form some of the key ingredients for reaching coordination and cooperation in a world of autonomous, even selfish entities. There's wealth of work considering both theoretical aspects of reputation and practical frameworks for implementing systems. The connections to the information retrieval-driven research in recommender and reputation systems are clear. It seems a very promising idea to combine these efforts. Agents can provide feedback on behalf of customers, extract useful recommendations from large data sets including inherent feedback information, reveal dishonest reporting by examining relationships between recommenders, offer platforms for open, distributed opinion management schemes, etc.

Thus, the various approaches for recommendation system have been discussed above. Now a day's reviews and comments on hotels on the web area are the important information source in travel planning. Therefore, knowing about these comments is important for quality control in any form of managerial association [16].Each comment is transferred into a feature-opinion pair and mining is then performed on the formatted comments utilizing the algorithm of association classification. The purpose for doing so is to centralize comments and summarize public opinions [17]. One related task of the opinion mining is to classify the opinions in different scales. In a number of cases, the purpose is to identify opinions in a text and classify them into positive, negative classes. The sentiment analysis when applied on those reviews and comments given by the users will give the "positive" or "negative" labelling [18].

Therefore, there is a need for a recommendation system or recommender system that behave as information filtering system that seeks to predict the 'rating' or 'preference' that user would give to an item. The recommendation system will study patterns of behaviour to know what someone will prefer from among a collection of things he has never experienced [19]. The recommender system reads thousands of reviews and rank entities like "movies', "hotels", and "restaurant" etc. according to some defined features and recommend the best entity in no time which is difficult to do manually as a person cannot read all the reviews.

Opinion Mining is one of the important branches of data mining which has been approached recently. Opinions can be thought as a combination of words, sentiments or documents, an extensive work on these factors can be found in [20], [21], [22] respectively. However, some people believe that opinion is topic dependant and the above methods do not have consideration for this [23]. The TREC (Text Retrieval Conference) came out with a Blog Track with a major task of information retrieval [24]. In the similar way, NTCIR set up an Opinion Analysis task with multi lingual test beds in Chinese, Japanese and English [25]. Mining product review is one of the major focuses for research in recent days [26]. There is a canvass on comparison for opinions [27]. Even the orientation on these features may differ significantly. e.g., "the picture quality of the camera is very good but strength of its body is very weak", here "picture quality" and "strength of body" is features. Both the features have different types of opinions, one is having positive and other is negative [28], [29], [30]. The reference [31] explores the clues for opinion mining and summarization. It makes use of lexicon-based method to find that expressed opinion for some specific product is positive or negative.

Customers' reviews are the basis for opinion mining technique. Finding and summarizing the opinion from huge amount of reviews from the customers, is also very tedious for business. For researchers, Opinion mining is a very important issue in the field of data mining. The main issue to consider is to find (a) Product feature and (b) Analysis comments, whether positive or negative [32], [33]. People generally use some pre-determined terms to interpret it as positive or negative comments, like, better, good, nice, well written, highly recommended etc. are treated as positive terms and worst, time consuming, bad, not recommended, etc. are termed as negative comments. Opinion retrieval is perceived as a two-step task, finding relevant documents and re-ranking 
these documents by opinion scores [23]. The reviews are given by the human and it is very evident that to understand the review one should perceive it as human being.

\section{APPROACHES FOR EVALUATION OF RECOMMENDATION SYSTEM}

Ranking plays an important part in the recommender system. To maximize the user utility the rated items are ranked and then recommended to users. The aim of ranking is to unearth the best possible ordering of a set of items for the user. To improve aggregate diversity of the recommender system several ranking techniques have been introduced which are discussed below. [8]

\subsection{Basic Approach}

In recommender systems it is the most commonly used approach for ranking the products. The ratings that are predicted by the system are ranked from highest to lowest.

$$
\operatorname{rank}_{\text {basic }}(p)=R \times(u, p)^{-1}
$$

Where $\mathrm{R} \times(\mathrm{u}, \mathrm{p})$ is the system's predicted rating. Products with highest predicted ratings that are recommended to user are those with the power of -1 . When this approach is implemented in recommender system it only increases accuracy not diversity.

\subsection{Product Popularity Based Approach}

In this approach ranking of product is based on the popularity of the product form lowest to highest. The popularity of the product is denoted by the number of users who have rated the product. When this approach is implemented in recommender system it increases diversity

$$
\operatorname{rank}_{\text {prodpop }}(p)=|U(p)|
$$

Where $|U(p)|=\{u \in U\} \ni R \times(u, p)$ and $\mathrm{R} \times(\mathrm{u}, \mathrm{p})$ is the known rating given by the user $\mathrm{u}$ to product $\mathrm{p}$.

\subsection{Backward Predicted Rating Approach}

The reverse of the Basic Approach is the Backward Predicted Rating Approach. The ratings that are predicted by the system are ranked from lowest to highest.

$$
\operatorname{rank}_{\text {BackPred }}(p)=R \times(u, p)
$$

\subsection{Product Absolute Likeability}

This approach ranks the product according to how many users liked the product.

$$
\operatorname{rank}_{\text {abslike }}(p)=|U H(p)|
$$

Where $U H(p)=\{u \varepsilon U(p) \mid R(u, p) \geq T H\}$

\subsection{Product Relative Likeability}

In this approach it ranks products according to the percentage of the users who really liked a product among all users who rated them.

$$
\operatorname{rank}_{\text {RelLike }}(p)=|U H(p)| /|U(p)|
$$

\section{CONCLUSIONS}

Recommender system is the one which takes user preferences and tastes on the basis of reviews and thus providing recommendations for the system. Due to the overload of information on the World Wide Web, the necessity of recommender systems to generate efficient solutions have evolved. Different approaches of recommender systems and techniques of ranking have been discussed in detail in this paper. In the present scenario, finding the right recommender for evaluating the credibility of recommender systems is an essential feature. Retrieval of information from huge volumes of data in diversified areas results in a tedious process. Hence, agent mediated recommender systems have evolved to make the recommendation process trivial and thereby implementing opinion mining for evaluating the various recommendation systems.

\section{REFERENCES}

[1] NanangHusin, "Internet User Behaviour Analysis in Online Shopping on Indonesia", proceedings of the 2011 international conference on Advanced Computer Science and Information System (ICACSIS) (Page: 137-142, ISBN: 978-1-4577-1688-1)

[2] Young Ae Kim, Jaideep Srivastava, "Impact of Social Influence in E-Commerce Decision Making" Proceedings of the ninth international conference on Electronic commerce.ICEC'07, (Pages 293-302, Year of publication: 2007, ISBN: 978-1-59593-700-1).

[3] K. Abhishek, S. Kulkarni, V. Kumar, N. Archana, P. Kumar, "A Review on Personalized Information Recommendation System Using Collaborative Filtering," "International Journal of Computer Science and Information Technologies (IJCSIT)", vol. 2, no. 3, pp. 1272-1278, 2011.

[4] J. Ben Schafer, Joseph Konstan, John Riedl, "Recommender systems in E-Commerce," Proceedings of the 1st ACM conference on Electronic commerce, (Page: 158-166, Year of publication: 1999, ISBN: 158113-176-3)

[5] Ziming Zeng. "An Intelligent E-Commerce Recommender System Based-on Web Mining" International Journal of Business and Management", Vol 4. No.7, 2009

[6] ManishaHiralall, "Recommender systems for e-shops, Business Mathematics and Informatics paper", Vrije Universiteit, Amsterdam. (2011)

[7] D. Jannach, M.Zanker, A.Felfernig, G.Friedrich. Recommender Systems: An Introduction. (Cambridge University Press, 2010)

[8] Antony Taurshia.A, S.Deepa Kanmani (2013) "Recommender System and Ranking Techniques: A Survey". International Journal of Engineering Research and Applications (IJERA) Vol. 3, Issue 1, pp.491-493, 2013

[9] S. Puntheeranurak, T. Chaiwitooanukool, "An Itembased Collaborative Filtering Method using Item Based Hybrid Similarity," proceedings of the IEEE 2nd International Conference on Software Engineering and Service Science (ICSESS), (Page: 469-472, Year of publication: 2011, ISBN: 978-1-4244-9699-0)

[10] M. Balabanovi, Y. Shoham, "Fab:contentbased,collaborative recommendation". "Magazine Communications of the ACM" Volume 40 Issue 3, pp.66-72, 1997

[11] Robin Bruke, Hybrid Web Recommender System, in the Adaptive Web, LNCS, Handbook of Methods and 
Strategies of Web Personalization (USA: Springer Berlin Heidelberg, 2007, 377-408)

[12] D. Sun, Z. Luo , F. Zhang, "A Novel Approach for Collaborative Filtering to Alleviate the New Item Cold Start Problem," proceedings of the 11th IEEE International Symposium on Communications and Information Technologies (ISCIT) ,(Pages: 402-406, Year of publication: 2011, ISBN: 978-1-4577-1294-4)

[13] Judy Kay, Scrutable Adaptation: Because We Can and Must, Handbook of Adaptive Hypermedia and Adaptive Web-Based Systems, (Ireland: Springer Berlin Heidelberg, 2006, 11-19).

[14] Alfarez Abdul Rahaman, Stephen Hailes, "Using Recommendations for Managing Trust in Distributed Systems", Proceedings of the IEEE International Conference on Communications.(Year of Publication: 1997)

[15] Olli Niinivaara, Agent-Based Recommender Systems, Software Agent Technology Course Paper, Department of Computer Science, University of Helsinki, 2004

[16] Walter Kasper, Mihaela Vela, "Sentiment Analysis for Hotel Reviews", in the Proceedings of the Computational Linguistics-Applications Conference. Jachranka, (Page: 45-52, Year of publication: 2011, ISBN: 978-83-6081047-7)

[17] Li-Chen Cheng, Zhi-Han Ke , Bang-Min Shiue, "Detecting changes of opinion from customer reviews", Proceedings of the Eighth International Conference on Fuzzy Systems and Knowledge Discovery (FSKD) Shanghai, (Page: 1798 - 1802, Year of publication: 2011, ISBN: 978-1-61284-180-9).

[18] AnanchaiMuangon, Thammaboosadee, S., Haruechaiyasak, C. " A Lexiconizing Framework of Feature-based Opinion Mining in Tourism Industry" Proceedings of the Fourth International Conference on Digital Information and Communication Technology and it's Applications (DICTAP), Bangkok, (Page: 169 - 173, Year of publication: 2014, ISBN: 978-1-4799-3723-3).

[19] EivindBjørkelund. Thomas H Burnett, Kjetil Norvag,"A Study of Opinion Mining and Visualization of Hotel Reviews" Proceedings of the 14th International Conference on Information Integration and Web-based Applications \& Services, Bali, Indonesia. (Page: 229 238, Year of publication: 2012, ISBN: 978-1-45031306-3)

[20] V. Hatzivassiloglou, K. McKeown, "Predicting the semantic orientation of adjectives" Proceeding of the ACL '98 Proceedings of the 35th Annual Meeting of the Association for Computational Linguistics and Eighth Conference of the European Chapter of the Association for Computational Linguistics archive, USA (Page: 174 181, Year of publication: 1997)

[21] Soo-Min Kim, Eduard Hovy, "Determining the sentiment of opinions" in the Proceedings of the 20th international conference on Computational Linguistics, Geneva (Page: 1367-1374, Year of publication: 2004)
[22] B. Pang, L. Lee, S. Vaithyanathan, "Thumbs up?: sentiment classification using machine learning techniques" Proceedings of the ACL-02 conference on Empirical methods in natural language processing (EMNLP), (Page: 79-86, Year of publication: 2002)

[23] Y. Fangy, L. Siy, N. Somasundaramy, Z. Yu, "Mining Contrastive Opinions on Political Texts using CrossPerspective Topic Model" Proceedings of the fifth ACM international conference on Web search and data mining (WSDM),Seattle, Washingtion, USA, (Page: 63 - 72, Year of publication: 2012, ISBN: 978-1-4503-0747-5 )

[24] I. Ounis, M. Rijke, C. Macdonald, G. Mishne, I. Soboro, "Overview of the TREC-2006 Blog Track," TREC, (Page: 15-27, Year of publication: 2006)

[25] Y. Seki, D. Evans, L. Ku, H. Chen, N. Kando, and C. Lin, " Overview of opinion analysis pilot task at NTCIR6," (Page: 265 - 278, Year of publication: 2007)

[26] X. Ding, B. Liu, P. Yu, "A holistic lexicon-based approach to opinion mining" ,Proceedings of the 2008 International Conference on Web Search and Data Mining (WSDM), (Page: 231 - 240, Year of publication: 2008, ISBN: 978-1-59593-927-2 )

[27] B. Liu, M. Hu, J. Cheng, "Opinion observer: Analyzing and comparing opinions on the web," Proceedings of the 14th international conference on World Wide Web (WWW), (Page: 342 -351, Year of publication: 2005, ISBN: 1-59593-046-9)

[28] V. Hatzivassiloglou, J. Wiebe, "Effects of adjective orientation and gradability on sentence subjectivity" Proceedings of the 18th conference on Computational linguistics (COLING), (Page: 299 - 305, Year of publication: 2000, ISBN: 1-55860-717-X)

[29] P. Beineke, T. Hastie, C. Manning, S. Vaithyanathan, "An Exploration of Sentiment Summarization," Proceedings of the AAAI Spring Symposium on Exploring Attitude and Affect in Text: Theories and Applications,(Year of Publication: 2003).

[30] N. Kaji, M. Kitsuregawa, "Automatic Construction of Polarity-Tagged Corpus from HTML Documents," Proceedings of the COLING/ACL on Main conference poster sessions, (Page: 452-459, Year of publication: 2006)

[31] M. Hu, B. Liu, "Mining and summarizing customer reviews," Proceedings of the tenth ACM SIGKDD international conference on Knowledge discovery and data mining (KDD) (Page: 168-177, Year of publication: 2004, ISBN: 1-58113-888-1)

[32] A.M. Popescu, O. Etzioni, "Extracting Product Features and Opinions from Reviews" Proceedings of the conference on Human Language Technology and Empirical Methods in Natural Language Processing (EMNLP) (Page: 339-346, Year of publication: 2005)

[33] L. Zhuang, F. Jing, X.-Yan Zhu, L. Zhang. "Movie Review Mining and Summarization", Proceedings of the 15th ACM international conference on Information and knowledge management (CIKM) (Page: 43 - 50, Year of publication: 2006, ISBN: 1-59593-433-2) 